\title{
SIMPLE SCREENING FOR POTENTIAL CHRYSENE DEGRADING FUNGI
}

\author{
Asep Hidayat ${ }^{1 *}$, and Sanro Tachibana ${ }^{2}$ \\ a. Forest Research and Development Agency (FORDA), Ministry of Forestry - Republic of Indonesia, \\ Manggala Wanabakti Building, Jl. Jend. Gatot Subroto, Jakarta - Indonesia \\ ${ }^{\mathrm{b}}$ Department of Applied Bioscience, Faculty of Agriculture, Ehime University 3-5-7 Tarumi, Matsuyama, Ehime, \\ 790-8566 Japan \\ E-mail address: ashephidayat@forda-mof.org
}

\begin{abstract}
Chrysene is a class of organic compounds, arranged in four benzene rings, and a polycyclic aromatic hydrocarbon $(\mathrm{PAH})$, It has been found to have a variety of toxicity, mutagenicity, teratogenicity, and carcinogenicity on microorganisms, plants and animals in environment. Nowadays, the most attention on degradation of PAHs is investigating degradation of high-molecular-weight molecules. However, microbes which have ability to degrade PAHs containing more than three benzene rings are more difficult to be obtained. In order to provide chrysene degrading fungi, this study was conducted for screening, and isolating the fungi from soil, and hence investigating the selected fungi having high chrysene degradation activity. From the 62 soil samples collected from Matsuyama-Japan, 92 isolates were found and 20 isolates of them grew well in Malt extract media contaminated with chrysene (covered up 90\%). Among them, a fungus, Fusarium sp. has the highest activity to degrade chrysene compared to others screened fungi. This fungus was evaluated further on liquid medium from distilled water and sea water to confirm their validity in degrading chrysene. The result showed that Fusarium sp. F092 degraded $48 \%$ of chrysene, where the chrysene degradation showed no differences at salinity of $35 \%$. The effect of variation of enzymes activities on incubation times was evaluated simultaneously. When the fungus was grown in a liquid culture, the highest activity of 1,2-dioxygenase reached $203.5 \mathrm{UL}^{-1}$ were observed on 30 days incubation and $29.7 \mathrm{Ul}^{-1}$ for 2,3-dioxygenase on 40 days incubation. The products of chrysene degradation by Fusarium sp. F092 are, 1-hydroxy 2-napthoic acid and catechol. In conclusion, Fusarium sp. F092 shows a high potential activity degrade PAHs contamination .
\end{abstract}

Key words: Screening, chrysene, bioegradation, Fusarium sp. F092

\section{INTRODUCTION}

Polycyclic aromatic hydrocarbons (PAHs), a large group of xenobiotic pollutans, are common, persistent and recalcitrant contaminants. Polycyclic aromatic hydrocarbons can be reduced from polluted soil by incineration, thermal desorption, and soil washing, but these methods are expensive and have negative effect to the environment. Another way is bioremediation as a competitive alternative to convert pollution into less toxic form.

Many varieties of microorganisms, such as bacteria, fungi, alga, show activity to detoxify pollutants materials (Cerniglia, 1992; Juhasz \& Naidu, 2000). A wide variety of fungi have also demonstrated ability to metabolize PAHs of two to six rings type molecules. The white rot fungi, Phanerochaete chrysosporium, Trametes versicolor, Pleurotus ostreatus, Bjekandera sp, and the brown-rot fungi Neolentinus lepideus were reported to be able to degrade various PAHs (Matsubara et al., 2006; Verdin et al., 2004). Other fungi such as non lignolytic fungi, Coniothyrium sp., Penicillilium sp., Cunninghamella elegrans, Trichoderma viride, Fusarium solani, Phialophora alba, have exhibited a significant potential to metabolize various PAHs to polar metabolites (Verdin et al., 2004; Potin et al., 2004; Ravelet et al., 2000). Non ligninolytic fungi are major group of fungi that metabolize various PAHs by cytochrome P450 monooxygenase, epoxide hydrolase and dioxygenase. Ligninolytic fungi are

ISSN 2413-0877 (C) 2015 The Authors.

Published by KnowledgeE Publishing Services This is an open access article under the CC BY-NC-ND license (http://creativecommons.org/licenses/by-nc-nd/4.0)

Selection and Peer-review under responsibility of the 3rd ICBS-2013

Doi http://dx.doi.org/10.18502/kls.v2i1.177 
fungi that can produce peroxidase and laccase type enzyme, which degrade PAHs by nonspecific radical oxidation.

Many investigations have focused on fungi which have ability to attack PAHs for cleaning up contaminated sites. The focus of PAH research in recent years on the degradation of high-molecular-weight PAHs has resulted in the isolation of a number of microorganisms that can mineralize and grow on four-ring PAHs as a sole carbon and energy source (Boonchan et al., 2000). Fungi capable of degrading PAHs containing more than four benzene rings are more difficult to be obtained. The aim of this research is to screen fungi from soil based on its ability to grow in chrysene agar medium, to evaluate the chrysene degradation activity of the isolated fungi in both saline and non saline liquid culture, and to investigate the enzyme activity secreted by selected fungi as well as its degradation pathway.

\section{MATERIALS AND METHODS}

\section{Chemical and microorganisms}

Chrysene, agar, glucose, wakogel S-1 silica gel and other chemical were purchased from Wako. Co. Ltd (Osaka, Japan). Thin layer chromatography (TLC) aluminium sheet (silica gel 60 F254, 20x20 cm) were obtained from Merck (Darmstadt, Germany). Synthetic of sea water was obtained from Delphis (Osaka, Japan). P. chrysosporium (NBCR31429) and T. vesicolor (NBCR30388) was obtained from National Biomedical Computation Resources (NBCR). P. ostreatus was obtained from Laboratory of Chemistry and Biotechnology for Utilization of Forest Resources, Ehime University, Japan.

\section{Sampling site and fungi isolation}

The soil samples were collected from several places in Matsuyama - Japan. Each soil sample was diluted three times with sterile water, $1 \mathrm{ml}$ of supernatant placed in a petri dish containing MEA supplemented with chloramphenicol $300 \mathrm{mgl}^{-1}$ and $1 \mathrm{mg}$ phenanthrene in the top layer. Fungi that grew on the medium were transferred to a fresh MEA medium to obtain pure strain. These isolates were selected based on their ability to grow well covering more than $90 \%$ of the petri dish area, and several fungi will be selected for further screening. They were tested again on MEA containing $1 \mathrm{mg}$ chrysene to obtain one fungus that is most capable to degrade chrysene and phenanthrene.

\section{Liquid culture condition}

The selected fungi were tested on liquid medium to confirm their activity in degrading chrysene. Liquid culture experiments were conducted in $100 \mathrm{ml}$ erlenmeyer flasks containing $20 \mathrm{ml}$ of malt extract (ME) liquid medium. Medium were sterilized for $20 \mathrm{~min}$ at $121^{\mathrm{Tm}} \mathrm{C}$ and then three disk ( $Æ 5 \mathrm{~mm}$ ) obtained by punching out with a cork-borer from an actively growing fungus in agar plate were inoculated into the erlenmeyer flask. After pre-incubation $1 \mathrm{mM}$ of chrysene was added to each inoculated flask and then incubated again for 15 and 30 days in the dark condition at $25{ }^{\mathrm{TM}} \mathrm{C}$. As effect of salinity, ME liquid medium was prepared by $35 \mathrm{gl}^{-1}$ of synthetic sea water. 


\section{Extraction of residual chrysene and chrysene metabolite}

Chrysene residues were quantified every 15 and 30 days. Liquid culture was extracted and purified by a chromatography column ( $3 \mathrm{~g}$ of $\mathrm{S} 1$ silica gel and anhydrous $\mathrm{Na}_{2} \mathrm{SO}_{4}$ ) eluted with $150 \mathrm{ml}$ dichloromethane. The concentrate was subjected to a gas chromatography coupled with mass spectrometer (GG-MS Shimadzu QP-5000), equipt with a TC-5 column (30 m, id: $0.24 \mathrm{~mm}$ ). The carrier gas was Helium at a constant flow rate of $1.5 \mathrm{ml} / \mathrm{min}$ with column pressure $100 \mathrm{Kpa}$ and interface temperature $260{ }^{\mathrm{TM}} \mathrm{C}$. The temperature program was started at $80{ }^{\mathrm{TM}} \mathrm{C}, 20{ }^{\mathrm{TM}} \mathrm{C} / \mathrm{min}$ to $150{ }^{\mathrm{TM}} \mathrm{C}, 25^{\mathrm{TM}} \mathrm{C} / \mathrm{min}$ to $300{ }^{\mathrm{TM}} \mathrm{C}$ which maintained for 10 min to allow late eluting peak to exit the column. The injection volume was $1 \mu$ and the injector temperature was maintained at $260{ }^{\mathrm{TM}} \mathrm{C}$. Chyesene metabolite was investigated by extraction, purified and analyzed. The metabolite and authentic compound were methylated with trimethylsilylation (TMS) prior to GC-MS analysis. The methylated metabolite and authentic compound were performed analyzed by gas chromatography coupled with mass spectrometer (GG-MS Shimadzu QP-5000), equipt with a TC-5 column (30 m, id: $0.24 \mathrm{~mm}$ ). The GCMS condition analysis consist a detector at $1.3 \mathrm{eV}$, scan interval (per-second) and mass range of $40-500$.

\section{Enzymes assay}

The enzyme activity in the culture medium was calculated based on spectrophotometry analysis using a Shimadzu UV-1600 spectrophotometer. 1,2-Dioxygenase activity was assayed by the method described by Nakazawa and Atsushi (Nakazawa \& Atsushi, 1970). 2,3-Dioxygenase activity was assayed by the method described by Nozaki (1970).

\section{RESULTS AND DISCUSSION}

\section{Screening and isolating fungi}

The 62 samples were collected from Matsuyama City- Japan. From these samples, 92 isolates were found and 20 isolates of them grow well and covered more than $90 \%$ of petri dish area containing $1 \mathrm{mg}$ of phenanthrene. Among them seven isolates shown highest ability to grow on agar containing phenanthrene were selected for further screening. Seven isolated fungi were evaluated for their growth ability on degrading chrysene to obtain one fungus that is most capable. Fig. 1 shows the growth capability of seven selected fungi grows on agar medium containing chrysene. The best fungi shows growth performance up to $72 \%$ and $77 \%$ were of isolate F055 and F092. Fungi F092 was the best compared with other tested fungi, and selected for further study. Identification using morphological character and molecular analysis showed that F092 was identical to those of Fusarium sp.

Several fungi available in our laboratory, P. chrysosporium, T. vesicolor, and P. ostreatus were chosen for growth comparison with fungi Fusarium sp. F092. The ability of fungi growth on agar medium from sea water with $35 \%$ of salinity was also investigated. The effectiveness of bioremediation is dependent on the ability of the natural micro flora to tolerate the level of salinity in the environment. In screening processes, Fusarium sp. F092 was adaptive growth on saline agar medium containing chrysene and the ability increases $20 \%$ compared to the non saline agar medium (Fig. 2). 


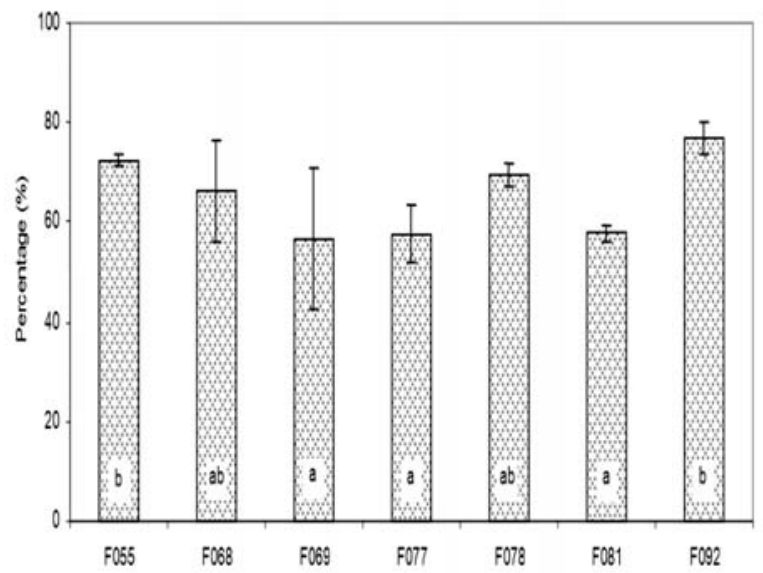

Figure 1. Growth of selected fungi on agar medium contaminated chrysene $(n=3)$. The bars followed by one or more same letters are not significantly difference $(\mathrm{P}<0.05)$

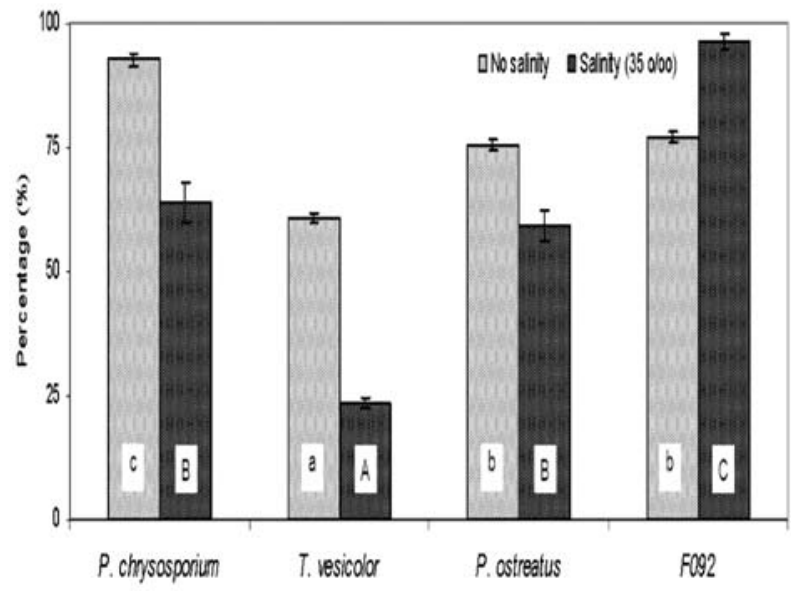

Figure 2. Growth of several fungi on agar medium containing chrysene $(n=3)$. The bars followed by one or more same letters are not significantly difference $(P<0.05)$

\section{Degradation of chrysene with Fusarium sp. F092 in a liquid medium}

Fig. 3 showed the degradation rate of chrysene in a liquid medium. The chrysene degradation rate for three fungi, $P$. chrysosporium, $P$. ostreatus and Fusarium sp. F092, were $38 \%, 26 \%$ and $42 \%$ for 15 days, and $52 \%, 45 \%$ and $48 \%$ for 30 days, respectively The degradation rate of chrysene by Fusarium sp. F092 is not significant different $(\mathrm{P}<0.05)$ with $P$. chrysosporium on 15 days and not significant different $(\mathrm{P}<0.05)$ both with $P$. chrysosporium and $P$. ostreatus on 30 days observation. As the effect of sea water salinity $(35 \%)$, the ability of Fusarium sp. F092 on chrysene degradation was significantly decrease $(\mathrm{P}<0.05)$ on 15 days and no significant decrease $(\mathrm{P}<0.05)$ on 30 days (Fig. 4). PAHs degradation by Fusarium strains was reported by Verdin, (2004). These results give new information that Fusarium sp. F092 is also able to degrade chrysene as it was only known for certain fungi that previously recorded (Matsubara, 2006; Juan et al., 2008; Harayama, 1997). More over, increased salinity decreases the degradation of the number or type of substrate and the ability of some microorganisms (Sing, 2006; Obuekwe, 2005). In addition, biodegradation by microorganisms in the present salinity was slow, because this environment tends to disrupt cell membrane, denature some proteins, change osmotic force which any of these situation could be lethal (Woolard \& Irvine, 1994; Kargi \& Dinçer, 2000).

\section{Evaluation of enzyme activity}

The enzyme activity during incubation of Fusarium sp. F092 in a liquid medium was investigated. The variation activities from one enzyme to another and in function of incubation time by Fusarium sp. F092 (Fig. 5), indicated that Fusarium sp. F092 degraded chrysene as carbon and energy source. When the fungus was grown in liquid culture from 10 to 40 days, the activity of 1,2-dioxygenase was higher than 2,3-dioxygenase. The highest activity of 1,2-dioxigenase reached $203.5 \mathrm{Ul}^{-1}$ on 30 days and $29.7 \mathrm{Ul}^{-1}$ for 2,3-dioxigenase on 40 days. In other liquid culture, Fusarium sp. F092 was detected to produce ligninolytic 
enzymes. Manganese peroxidase and laccase activities reached 49.6 and $18.5 \mathrm{Ul}^{-1}$ on 20 day, respectively (not shown). The enzymes play a prominent role in chrysene degradation. Under non saline condition 1,2-dioxygenase activity was higher than that of 2,3-dioxygenase activity. 1,2-dioxygenase and 2,3-dioxygenase activity under non saline condition were higher than those on sea water. Salinity characteristic of sea water had minimum effect on ligninolytic activity of $I$. lacteus and $L$. tigrinus, while in $B$. adusta, activity was inhibited by salinity level of $32 \%$ (Valentín, 2006)

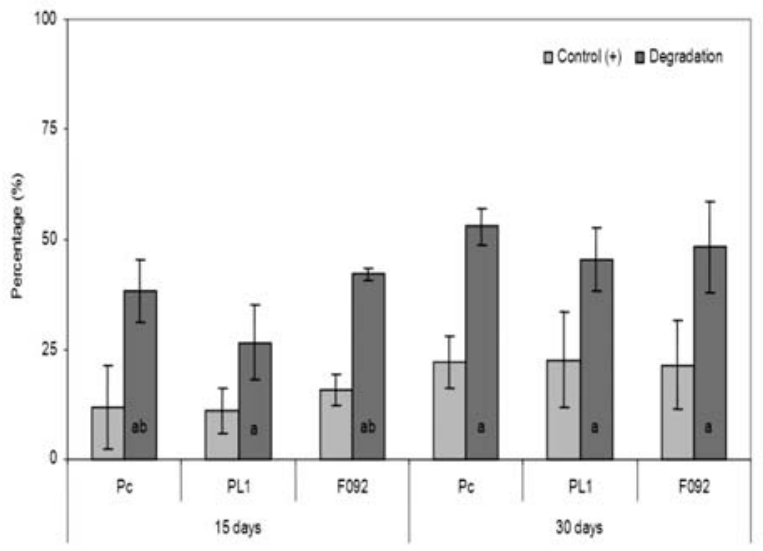

Figure 3. Degradation of chrysene with Fusarium sp. F092 in a liquid medium $(n=3)$. The bars followed by one or more same letters at the same time are not significantly difference $(\mathrm{P}<0.05) . \mathrm{Pc}=P$. chrysosporium; $\mathrm{PL1}$ $=P$. ostreatus.

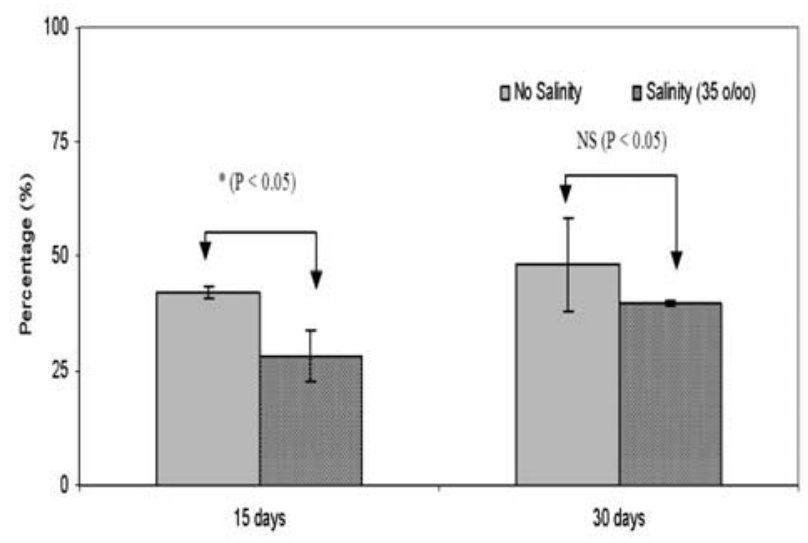

Figure 4. Effect of salinity on chrysene degradation by Fusarium sp. F092.

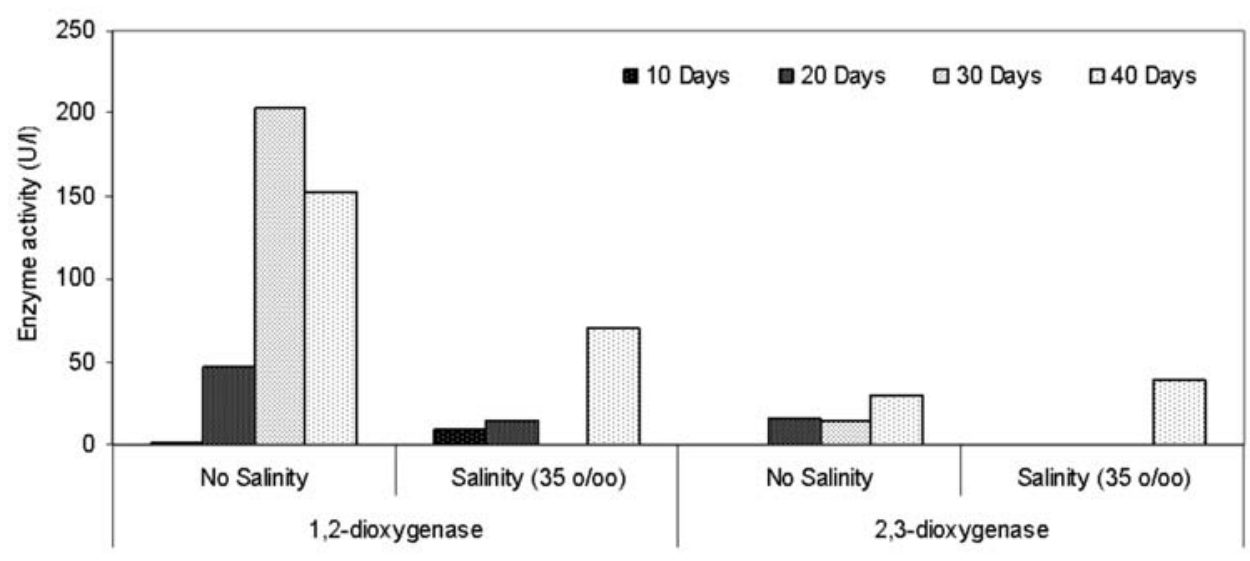

Figure 5. Change in enzyme activity during incubation of Fusarium sp. F092 in liquid medium

\section{Identification of chrysene metabolite}

After incubation of Fusarium sp. F092 in liquid medium for 15 days, compound I was isolated which had retention time $(R t)$ at $16.406 \mathrm{~min}$ and a molecule ion $\left[\mathrm{M}^{+}\right]$at $m / z 188$ with fragment ion at $m / z 170\left[\mathrm{M}^{+}-18\right]$ and $m / z 142\left[\mathrm{M}^{+}-28\right]$, representing probable losses of $\mathrm{H}_{2} \mathrm{O}$ and $\mathrm{CO}$. The mass spectral fragmentation pattern of the trimethysilylated (TMS) had a molecule ion $\left[\mathrm{M}^{+}\right]$at $M / z 332$ and fragment ion at $m / z 317\left[\mathrm{M}^{+}-15\right]$ and $m / z 259\left[\mathrm{M}^{+}-73\right]$, 
representing probable losses $-\mathrm{CH} 3$ and $-\mathrm{Si}\left(\mathrm{CH}_{3}\right)_{3}$. This mass spectral fragmentation pattern of isolated I and TMS isolated I suggested that this metabolites was 1-hydroxy 2-napthoic acid coincided with authentic standard.

After incubation of Fusarium sp. F092 in liquid medium for 30 days, compound (II) was isolated which had Rtat 7.746 min and a molecule ion [M+] at $m / z 110$ with fragment ion at $m /$ $z 92\left[\mathrm{M}^{+}\right.$-18], representing probable losses of $\mathrm{H}_{2} \mathrm{O}$ (Table 1). The mass spectral fragmentation pattern of the TMS had a molecule ion $\left[\mathrm{M}^{+}\right]$at $M / z 254$ and fragment ion at $m / z 239\left[\mathrm{M}^{+}\right.$ -15] and $m / z 181\left[\mathrm{M}^{+}-73\right]$, representing probable losses $-\mathrm{CH}_{3}$ and $-\mathrm{Si}\left(\mathrm{CH}_{3}\right)_{3}$. The mass spectral fragmentation of isolated II and TMS isolated II was identical to those of authentic catechol.

The data on identified metabolites have been used to propose a degradation pathway for chrysene. The data on identified metabolites, Fusarium sp. F092 would convert chrysene trans-1,2-dihydrodiol became 1-hydroxy 2-napthoic acid. The resultants dihydrodiol is then sequentially attacked by dehydrogenase and meta-cleavage enzyme. These reaction are considered to yield 1-hydroxy 2-napthoic acid (Pinyakong et al., 2000; Kiyohara et al., 1994). These finding suggested that the same of the enzyme could convert chrysene via intial dioxygenation at 1,2 and 2,3-position to obtain isolated compound I. Fusarium sp. F092 could also convert 1-hydroxy 2-napthoic acid undergoes oxidative to 1,2 dihydroxynaphthalene, which is further metabolized via salicylic acid. Salicylic acid could also be further converted via the formation of catechol after the aldose and hydroxylase reaction, tran-o-hydroxy benzylidene pyruvate (tHBPA) which further mineralized to carbon dioxide and water. In our knowledge, this report is the first which Fusarium species could degrade chrysene. This fungus are also involved in the degradation of chrysene with identified several chrysene metabolites product. In general, the catabolism of chrysene by Fusarium sp. F092 is metabolized by initial dioxygenation at 1,2-position.

\section{REFERENCES}

Cerniglia, C.E. 1992. Biodegradation. $3:$ 351-368.

Harayama, S. Curr. Opin. 1997. Biotechnol. 8 : 268-273.

Juan, D., C. Jun, Z. Juan, and G. Shixiang. 2008. J. Environ. Sci. 20: 88-93.

Juhasz, A., R. Naidu. 2000. Int. Biodeter. Biodegr. 45: 57-88.

Kargi, F., A.R. Dinçer. 2000. Water Environ. Res. 66:230-235.

Kiyohara, H., S. Torigoe, N. Kaida, T. Asaki, T. lida, H. Hayashi, and N. Takizawa. 1994. Bacteriol. 176 2439-2443.

Matsubara, M., J.M. Lynch, F.A.A.M. De Leij. 2006. Enzyme Microb. Technol. 39:13651372.

Nakazawa, T., N. Atsushi. 1970. Methods Enzymol. 17: 518-522.

Nozaki, M. 1970. Methods Enzymol. 17: 522-525.

Obuekwe, C.O., A.M. Badrudeen, E. Al-Saleh, J.L. Mulder. 2005. Int. Biodeter. Biodegr. 56 197-205.

Pinyakong O., H. Habe, N. Supaka, P. Pinpanichkarn, K. Juntongjin, T. Yoshida, K. Furihata, H. Nojiri, H. Yamane, T. Omori. 2000. FEMS Microbiol. Lett. 191 : 115-121.

Potin, O., C. Rafin, E. Veignie. 2004. Int. Biodeter. Biodegr. 54 : 45-52. 
Ravelet C., S. Krivobok, L. Sage, R. Steiman. 2000. Chemosphere. 40 : 557-563.

Sing, H. 2006. Mycoremediation: fungal bioremediation. John Wiley \& Sons, Inc.

Valentín, L., G. Feijoo, M.T. Moreira, J.M. Lema. 2006. Int. Biodeter. Biodegr. $58: 15-21$.

Verdin, A., A.L.S. Sahraoui, R. Durand. 2004. Int. Biodeter. Biodegr. 53 : 65-70.

Woolard, C.R., R.L. Irvine. 1994. Water Environ. Res. 66 : 230-235. 